\title{
Dissolved Oxygen Prediction Model of Crab Culture Industry Based on fuzzy neural network
}

Ting Li

Wuhan polytechnic university

Corresponding author

ABSTRACT: This article proposes a dissolved oxygen prediction model for water quality about aquaculture to solve the problems like low accuracy and poor robustness of traditional prediction methods about water quality based on principal component analysis (PCA), fuzzy neural network (FNN), and differential evolution combined with BP algorithm (DEBP).

KEYWORD: water quality of aquaculture; dissolved oxygen prediction; FNN

\section{INTRODUCTION}

Dissolved oxygen is a very important water quality indicator about fishery water, the condition of dissolved oxygen has great effect on water quality and the growth of cultured species. At present, the identification of dynamic changes of dissolved oxygen mostly adopts fixed-point and timing measurement and is mainly identified by managerial staff through observing the activity changes of living things bred in pond. This afterwards control usually has bad effect on the growth and development of cultured species. Therefore, before lack of oxygen, timely mastering the rules of dynamic changes of dissolved oxygen in the pond and making predictions about dissolved oxygen are the imperative problems needs to be solved in the process of aquaculture.

\section{PRINCIPLE COMPONENT ANALYSIS}

The main steps of PCA algorithm are as followed:

(1) Standardization of Original Data

To eliminate the effect due to the different dimension and large difference in numerical value of original data, it is necessary to standardize thefunctional formula of original data(1)

$x_{i j}{ }^{*}=\frac{x_{i j}-\overline{x_{j}}}{s_{j}} \quad i=1,2, \ldots, n ; \quad j=1,2, \ldots, p$

In this formula: $i$ is the sample number, $j$ is sample component $x_{i j}$ is the $j$-th component of the $i$-th sample, $x_{j}$ and $s_{j}$ respectively are the average value and standard deviation of the $j$-th variable.

$$
x=T L^{T}+E=T_{1} L_{1}^{T}+T_{2} L_{2}^{T}+\ldots+T_{q} L_{q}^{T}+E
$$

(2) Form the original data matrix $X=[X 1, X 2, \ldots, X p]$, through orderly arranging the processed variables, so that covariance matrix $S$ of matrix $X$ can be got. The characteristic root of matrix $S$ can be arranged in order $\lambda 1 \geq \lambda 2 \geq \lambda 3 \geq \lambda p \geq 0$, and its corresponding loading matrix is $L=[L 1, L 2, \ldots, L p]$. Decompose the matrix $X$ as outer product of principle component score matrix $T$, loading matrix $L$ and plus residual $\mathrm{E}$, which is:

$x=T L^{T}+E=T_{1} L_{1}^{T}+T_{2} L_{2}^{T}+\ldots+T_{q} L_{q}^{T}+E$

(3) Calculate the variance contributions. variance contribution:

$\eta=\frac{\sum_{i=1}^{q} \lambda_{i}}{\sum_{j=1}^{p} \lambda_{j}}>85 \%$

Choose $q$ principle components which have at least $85 \%$ of accumulative contribution among $p$ sample components.

\subsection{FNN Model}

This article adopts improved fuzzy neural networks to build the prediction model of dissolved oxygen in the culture pond. The improved FNN is a network of 5 layers that respectively are input layer, fuzzy layer, inference layer, fuzzy normalized layer and output layer. As shown in Figure $1^{[14]}$, variables $\{\mathrm{x} 1, \mathrm{x} 2, \mathrm{x} 3\}$ in the input layer are corresponding to 3 principle 
components achieved after the principle component analysis, that are $\mathrm{pH}$ value of water in the culture pond, ammonia nitrogen value and water temperature. And the output layer is dissolved oxygen in the culture pond.

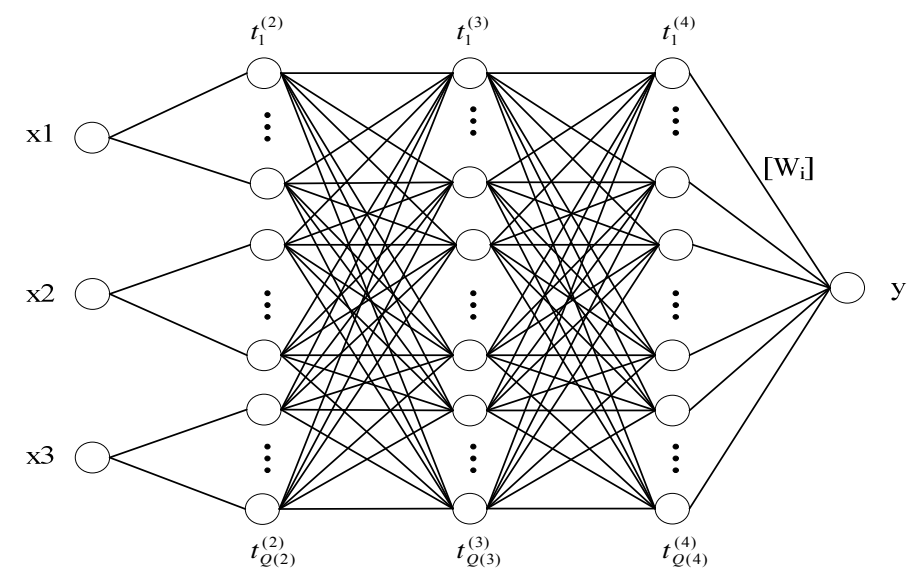

Figure 1.Fuzzy Neutral Network Model

Set the nodes number of input, output and the layer where it belongs of the j-th neuron unit of the k-th layer respectively as $S_{j}^{(k)}, t_{j}^{(k)}, U^{(k)}$.

The first layer is input layer, which shows the linguistic variables of input variables $(\mathrm{pH}$ value, ammonia nitrogen value and water temperature) $x 1, x 2, x 3$, and the input layer has not weighting relations with the second layer and directly delivers the input to the second layer.

Node activation function is:

$t_{i}^{(1)}=S_{i}^{(1)}=X_{i}, \mathrm{i}=1,2, \ldots, \mathrm{u}(1)$

The neuron number of the first layer is $U(1)=3$, with 3 corresponding principle components.

The second layer is fuzzy layer, composed of nodes of membership function with 21 neurons which show total fuzzy sets of input linguistic variables and complete the mapping from accurate input value to fuzzy value. Gaussian function has better smoothness than other membership functions, therefore choose it as membership function (5), and output membership values.

$h_{j}=\exp \left[-\frac{\left(x_{i}-c_{i j}\right)^{2}}{\mathrm{~b}_{j}{ }^{2}}\right], \mathrm{i}=1,2, \ldots, \mathrm{m}, \mathrm{j}=1,2, \ldots, \mathrm{u}$

In this formula, $C_{\mathrm{ij}}$ is the mean value of the $\mathrm{i}$-th Gaussian function of the $\mathrm{j}$-th neuron; $b_{j}$ is the variance of the i-th Gaussian function of the $\mathrm{j}$-th neuron; $C_{\mathrm{ij}}$ and $b_{j}$ are adjustable parameter sets.

The output of the $\mathrm{j}$-th neuron:

$\left.S_{j}^{(2)}=\mathrm{w}_{i j}^{(2)} t_{i}^{(1)}, t_{j}^{(2)}=h j\right\}, j=1,2, \ldots, \mathrm{u}(2), p=1, \ldots, \mathrm{m}_{\mathrm{i}}$ $\mathrm{w}_{i j}^{(2)}$ is the connection weight coefficient and the value is 1 , set $\mathrm{m}_{\mathrm{i}}$ as the number of fuzzy partitions of $\mathrm{x}_{\mathrm{i}}$, and then the nodes number of the second layer is $U(2)=\sum m_{i}$, the input variables of this article, $x 1, x 2, x 3$ are all divided into 7 fuzzy subsets, so $U(2)=21$.

The third layer is fuzzy inference layer, every neural junction means a fuzzy rule and its role is to match fuzzy rules, calculate the applicability of each rule and realize the fuzzy inference process.

\section{CONCLUSION}

Dissolved oxygen is a key parameter in pond aquaculture environment which can precisely predict the dissolved oxygen in the pond and is very meaningful for aquaculture. This article proposes to predict the dissolved oxygen through FNN and practice the parameters of FNN based on EDBP, which can avoid FNN training entering the local minimum and improve the accuracy of prediction. Compared with traditional BP neural network, the model built in this article has higher accuracy and is able to meet the actual demand about dissolved oxygen adjustment in shrimp culture industry, besides, it provides reference data for management and prediction of water quality in other fields.

\section{REFERENCES}

[1] GuanChong.Wu Liu Huang, Hongqiao Song. The role of increasing oxygen machine in shrimp farming[J].Transactions of the CSAE .2012.28(9):208-212.

[2] Jian Gu.Haitao Gu.TaoMen. Several mechanical way of increasing oxygen in aerobic performance comparison of pond farming. Transactions of the CSAE.2011.27 (1):148-152.

[3] Ying Zhang. Qianqian Gao Comprehensive water quality model and the fuzzy neural network research Based on the grey prediction model[J].Chinese Journal of Environmental Engineering.2015.9(2):537-545. 